\title{
ESPÉCIES DE CUPINS (ISOPTERA) EM CULTURA DE EUCALIPTO SOB DIFERENTES SISTEMAS DE MANEJO DE IRRIGAÇÃO, EM REGIÃO DE TRANSIÇÃO CERRADO-PANTANAL DE MATO GROSSO DO SUL, BRASIL ${ }^{1}$
}

Ana Paula Tavares da Silva², Hélida Ferreira da Cunha ${ }^{3}$, Jorge Adriano De Deus Ricardo ${ }^{4}$ e Alfredo Raúl Abot ${ }^{5}$

\begin{abstract}
RESUMO - O eucalipto sofre severos danos por cupins durante a implantação das mudas no campo, sendo considerada praga importante da cultura. No ecótono Cerrado/Pantanal não existem estudos anteriores sobre a ocorrência de cupins em plantações de eucalipto. O objetivo foi conhecer a riqueza de cupins, sua distribuição nos tratamentos e a influência do ambiente nas populações de cupins, nos híbridos de eucalipto Eucalyptus grandis x E. camaldulensis e Eucalyptus urophylla x E. grandis com fertirrigação por gotejamento, microaspersão e sequeiro. O delineamento experimental foi o de blocos ao acaso, com 10 tratamentos e quatro repetições. A riqueza de cupins foi associada pela Análise de Correspondência e pela influência climática pela Análise de Componentes Principais. Os cupins foram coletados de março de 2012 a fevereiro de 2013, em 432 parcelas de 4,0 x 2,25 m, em uma área de 3 ha, seguindo o protocolo rápido de coleta, observando-se a serrapilheira, e até $30 \mathrm{~cm}$ de profundidade no solo. Obtiveram-se 18 espécies de Termitidae, Rhinotermitidae, Heterotermitinae, Syntermitinae, Apicotermitinae e Nasutitermitinae. Nos tratamentos com gotejamento, houve a maior frequência total, com presença de 18 espécies de cupins nos híbridos, sem associação significativa. A associação entre espécies de cupins e tratamentos constituiu quatro grupos. A precipitação e a umidade relativa do ar influenciaram significativamente na comunidade dos cupins. Portanto, a maior riqueza de espécies ocorreu nos tratamentos com gotejamento. Das espécies consideradas pragas de eucalipto, destacou-se Syntermes molestus, pela sua maior frequência durante as coletas. Ocorreu diferença significativa na distribuição das espécies de cupins associadas aos tratamentos entre os meses de coleta. A riqueza e a distribuição dos cupins são influenciadas ao longo do ano pelos diferentes manejos de irrigação em áreas de cultivo de eucalipto.
\end{abstract}

Palavras-chave: Eucaliptocultura; Formigas-brancas; Térmitas.

\section{TERMITE SPECIES (ISOPTERA) IN EUCALYPTUS CULTURE UNDER DIFFERENT IRRIGATION MANAGEMENT SYSTEMS, IN A REGION OF TRANSITION FROM CERRADO TO PANTANAL IN MATO GROSSO DO SUL, BRAZIL}

\begin{abstract}
Eucalyptus suffers severe damage by termites during deployment of seedlings in the field, and it is an important pest of the crop. In the ecotone Cerrado/Pantanal there are no previous studies on the occurrence of termites in eucalyptus plantations. The objective was to know the richness of termites, their distribution in treatments and the influence of the environment on the population of termites in Eucalyptus hybrid Eucalyptus grandis $x$ E. camaldulensis and Eucalyptus urophylla $x$ E. grandis with fertigation by dripping, micro sprinkler and only rain (dryland). The experimental design was a randomized block design with 10 treatments and four replications. The richness of termites was associated by Correspondence Analysis and climate influence by Principal Component Analysis. Termites were collected from March 2012 to February 2013, in 432 plots of $4.0 \times 2.25 \mathrm{~m}$, in an area of 3 ha, following the rapid protocol for collection, observing
\end{abstract}

\footnotetext{
${ }^{1}$ Recebido em 05.02.2014 aceito para publicação em 10.11.2014

${ }^{2}$ Universidade Estadual de Mato Grosso do Sul, Programa de Pós-Graduação em Agronomia, Produção Vegetal, Aquidauna, MS - Brasil. E-mail: <agro_tavares@hotmail.com>.

${ }^{3}$ Universidade Estadual de Goiás, Departamento de Zoologia, Anápolis, GO - Brasil. E-mail: <cunhahf@gmail.com>.

${ }^{4}$ Universidade Estadual de Mato Grosso do Sul, Laboratório de Entomologia,Aquidauana, MS - Brasil. E-mail:<agro_tavares@hotmail.com>.

${ }^{5}$ Universidade Estadual de Mato Grosso do Sul, Aquidauana, MS - Brasil. E-mail: <arabot@uems.br>.
}

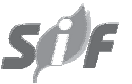

Revista Árvore, Viçosa-MG, v.39, n.1, p.137-146, 2015 http://dx.doi.org/10.1590/0100-67622015000100013 
the litter and up to $30 \mathrm{~cm}$ into the ground. 18 species were obtained of Termitidae, Rhinotermitidae, Heterotermitinae, Syntermitinae, Apicotermitinae and Nasutitermitinae. In treatments with dripping there was the highest total frequency with the presence of 18 species of termites in the hybrids, and no significant association. The association between termite species and treatments constituted four groups. Rainfall and relative humidity significantly influenced the termites community. Therefore, the highest species richness occurred in treatments with dripping. Among the species considered as pests of eucalyptus, Syntermes molestus stood out by their greater frequency during collections. There was a significant difference in the distribution of termite species associated with treatments between the months of collection. The richness and the distribution of termites are affected throughout the year by different irrigation managements on plantations of eucalyptus.

Keywords: Eucalyptus culture; White-ants; Termites.

\section{INTRODUÇÃO}

O gênero Eucalyptus pertence à família Myrtaceae e desenvolve-se satisfatoriamente em diversas situações edafoclimáticas (SANTOS et al., 2001).

No Brasil, o eucalipto teve seu plantio intensificado no século XX, para fornecer material para a Companhia Paulista de Estradas de Ferro. Assim, a crescente demanda por produtos de origem florestal têm favorecido o surgimento de insetos-praga, em função da redução da complexidade biológica (PAES, 2002).

O eucalipto sofre danos severos provocados por cupins durante a implantação das mudas no campo. A ocorrência de plantas jovens de eucalipto danificadas por térmitas foi relatada por Varma e Swaran (2007) na Índia, em estudo com o uso de transecto. Foram coletadas 11 espécies de duas famílias e quatro subfamílias. Nair e Varma (2003) observaram que as maiores perdas ocorreram em cultivos de quatro a seis meses após a implantação no campo.

Plantas adultas também podem sofrer ataques por térmitas. Sales et al. (2010) verificaram danos por 21 espécies de duas famílias e 16 gêneros de cupins no cerne de plantas adultas na Bahia. Duas espécies de Nasutitermes ultrapassaram o limite de dominância. Esses autores citaram que as extensas áreas cultivadas com eucalipto em algumas regiões da Bahia podem ter favorecido a dominância dos xilófagos por serem menos suscetíveis aos distúrbios ambientais e porque o eucalipto oferece abundância de alimento.

Dessa forma, os cupins são considerados pragas importantes na cultura do eucalipto. Na região do ecótono Cerrado/Pantanal são desconhecidas as espécies de cupim que ocorrem na cultura, como também as perdas que esses insetos causam nos reflorestamentos com eucalipto.
Há registros de danos de cupins em eucalipto nas mais diversas regiões do mundo (PERALTA et al., 2004; SILVA et al., 2004; JUNQUEIRA et al., 2008). No ecótono Cerrado/Pantanal não havia pesquisas sobre as espécies de cupins ocorrentes em plantação de eucalipto.

Os objetivos deste estudo foram (i) conhecer a fauna de cupins ocorrente em área de cultivo de eucalipto, híbridos de Eucalyptus grandis $\mathrm{x}$ E. camaldulensis e Eucalyptus urophylla $\mathrm{x}$ E. grandis, com diferentes tipos de irrigação; (ii) relacionar as espécies de cupins com os diferentes formas de irrigação; e (iii) determinar a influência da precipitação pluviométrica, umidade relativa do ar e temperatura sobre o comportamento das espécies de cupins.

\section{MATERIAL E MÉTODOS}

A pesquisa foi realizada na fazenda experimental da Unidade Universitária de Aquidauana, da Universidade Estadual de Mato Grosso do Sul (UEMS) $\left(20^{\circ} 28^{\prime} \mathrm{S} / 55^{\circ} 48^{\prime} \mathrm{W}\right.$; altitude de $\left.149 \mathrm{~m}\right)$. Segundo a classificação de Köppen, o clima é do tipo Aw (tropical quente - úmido), com verão chuvoso e inverno seco, com precipitação anual de 1.250 a $1.500 \mathrm{~mm}$ e temperatura média anual de $26^{\circ} \mathrm{C}$. A composição botânica da região compreende extensas áreas de pastagens cultivadas com Urochloa (= Brachiaria) spp. e pomares no contexto da agricultura familiar próximos a encostas de serra. Antes da implantação do eucalipto, a área era cultivada com Urochloa brizantha (Stapf) Webster cv. Marandu.

As coletas foram realizadas de março de 2012 a fevereiro de 2013, em área de eucalipto cultivada com os híbridos grancam (Eucalyptus grandis Hill $\mathrm{x}$ Eucalyptus camaldulensis Dehnh) (H1) e urograndis (Eucalyptus urophylla Blake x Eucalyptus grandis) (H2) plantados com distribuição espacial de 2,25 m

Revista Árvore, Viçosa-MG, v.39, n.1, p.137-146, 2015 
entre plantas e $4 \mathrm{~m}$ entre linhas, manejados com fertirrigação por gotejamento (G) e microaspersão (M) e sistema de sequeiro (S), com adubação manual.

O plantio das mudas de eucalipto foi realizado no dia 20 de abril de 2011. Após o plantio, as mudas foram adubadas conforme recomendações de Andrade (2004), a partir da análise química do solo, em que foram distribuídos $115 \mathrm{~g}$ de adubo formulado 04-20-20 por cova. Trinta dias após o plantio, as plantas mortas foram substituídas.

O delineamento experimental foi o de blocos ao acaso com 10 tratamentos. Estes consistiram em (i) microaspersão com o híbrido grancam + adubação convencional (MH1C); (ii) microaspersão com o híbrido urograndis + adubação convencional (MH2C); (iii) microaspersão com o híbrido grancam + fertirrigação (MH1F); (iv) microaspersão com o híbrido urograndis + fertirrigação (MH2F); (v) gotejamento com o híbrido grancam + adubação convencional (GH1C); (vi) gotejamento com o híbrido urograndis + adubação convencional (GH2C); (vii) gotejamento com o híbrido grancam + fertirrigação (GH1F); (viii) gotejamento com o híbrido urograndis + fertirrigação (GH2F); (ix) sequeiro com o híbrido grancam + adubação convencional (SH1C); e ( $x$ ) sequeiro com o híbrido urograndis + adubação convencional (SH2C) com quatro repetições, representadas pelos blocos, exceto no sistema de sequeiro, em que foram utilizadas duas repetições.

Foram realizadas coletas mensais nas áreas de cada híbrido, em que as parcelas foram representadas pelas áreas contendo quatro plantas de eucalipto em cada um dos tratamentos.

A amostragem em cada parcela foi realizada no tronco das arvores até $2 \mathrm{~m}$ de altura. No solo, foi observada a possível presença de cupins na serrapilheira e, posteriormente, no solo, em escavações de até 30 $\mathrm{cm}$ de profundidade, conforme metodologia proposta por Jones e Eggleton (2000). O tempo de amostragem foi de15 min por parcela/pessoa, como utilizado por Oliveira et al. (2008). Os cupins coletados foram acondicionados em frascos com etanol $80 \%$, com registro em caderneta de campo e encaminhados para o laboratório para identificação até o nível de gênero. A identificação em nível de espécie foi realizada por membros da equipe, especialistas nessa ordem de insetos. Amostras dos indivíduos utilizados na identificação encontram-se depositadas no acervo do Laboratório de Ecologia da
Universidade Estadual de Goiás (UEG) e de Entomologia da Universidade Estadual de Mato Grosso do Sul (UEMS).

Para conhecer a associação das espécies com os tratamentos, os resultados foram submetidos à Análise de Correspondência (AC), enquanto para estabelecer a influência dos fatores climáticos sobre as espécies de cupins, os tratamentos foram submetidos à Análise de Componentes Principais (ACP) (MAROCO, 2007), com o auxílio do software PAST versão 3.0 (HAMMER et al., 2013).

\section{RESULTADOS}

Foram coletadas 1.719 amostras de cupins nos três sistemas de manejo, obtendo-se 18 espécies, pertencentes a duas famílias e 11 gêneros (Tabela 1). Nas áreas dos tratamentos com gotejamento, em ambos os híbridos foram encontradas todas as espécies coletadas no decorrer do experimento. Nesse sistema de irrigação aconteceu a maior frequência total de cupins (Tabela 2). Não houve influência significativa do sistema de aplicação do fertilizante e dos híbridos sobre a comunidade de cupins. A espécie Diversitermes sp. (10) somente foi encontrada em GH1. Labiotermes longilabius (14) e L. orthocephalus (13) foram coletados apenas em GH2. O sistema de sequeiro com o híbrido urograndis teve 10 espécies, representando o menor número de ocorrências nas coletas. As espécies L. laticephalus (12) e Syntermes nanus (17) não foram observadas no sistema de sequeiro, e no sistema de microaspersão não foram coletados indivíduos de Heterotermes longiceps (11) e Grigiotermes sp. (7).

A análise de correspondência (AC) permitiu obter duas dimensões que explicaram $53 \%$ da inércia total, sendo $29 \%$ no eixo 1 e $24 \%$ no eixo 2 . Foi possível observar associação significativa das espécies com os sistemas de manejo da irrigação $\left(\chi^{2}=205,94\right.$; $\mathrm{p}=0,03)$ (Figura 1).

$\mathrm{O}$ tratamento $\mathrm{GH} 2$ teve destaque em relação com o eixo 1 e o eixo 2, que representa a associação das espécies com os tratamentos GH1, SH1 e SH2. A associação entre as espécies e os tratamentos permitiu a formação de quatro grupos diferentes. O grupo 1 (Anoplotermes sp. 1 (1), Anoplotermes sp. 2 (2), Anoplotermes sp. 3 (3) e Anoplotermes sp. 4 (4) não tiveram especificidade em relação a determinado tratamento, explicado pela proximidade com o centroide.

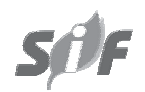

Revista Árvore, Viçosa-MG, v.39, n.1, p.137-146, 2015 
Tabela 1 - Famílias/espécies e grupos tróficos de cupins amostrados em área cultivada com eucaliptos, híbridos grancam (H1) e urograndis (H2), no período de março de 2012 a fevereiro de 2013, no Município de Aquidauana, MS.

Table 1 - Families/species and trophic groups of termites sampled in an area cultivated with eucalyptus, hybrid grancam (H1) and urograndis (H2), from March 2012 to February 2013, in the municipality of Aquidauna, MS.

\begin{tabular}{|c|c|c|c|c|c|c|}
\hline \multirow{3}{*}{ Famílias/Espécies } & \multicolumn{6}{|c|}{ Sistemas } \\
\hline & \multicolumn{2}{|c|}{ Microaspersão } & \multicolumn{2}{|c|}{ Gotejamento } & \multicolumn{2}{|c|}{ Sequeiro } \\
\hline & H1 & $\mathrm{H} 2$ & $\mathrm{H} 1$ & $\mathrm{H} 2$ & $\mathrm{H} 1$ & $\mathrm{H} 2$ \\
\hline \multicolumn{7}{|l|}{ TERMITIDAE } \\
\hline \multicolumn{7}{|l|}{ Apicotermitinae } \\
\hline 1 Anoplotermes sp. 1 & $\mathrm{x}$ & $\mathrm{x}$ & $\mathrm{x}$ & $\mathrm{x}$ & $\mathrm{x}$ & $\mathrm{x}$ \\
\hline 3 Anoplotermes sp. 3 & $\mathrm{X}$ & $\mathrm{X}$ & $\mathrm{X}$ & $\mathrm{x}$ & $\mathrm{x}$ & $\mathrm{x}$ \\
\hline 4 Anoplotermes sp. 4 & $\mathrm{x}$ & $\mathrm{x}$ & $\mathrm{x}$ & $\mathrm{x}$ & $\mathrm{x}$ & $\mathrm{x}$ \\
\hline 5 Anoplotermes sp. 5 & & & $\mathrm{x}$ & $\mathrm{x}$ & $\mathrm{x}$ & \\
\hline 6 Aparatermes sp. & $\mathrm{x}$ & $\mathrm{x}$ & $\mathrm{x}$ & $\mathrm{x}$ & $\mathrm{x}$ & $\mathrm{x}$ \\
\hline 7 Grigiotermes sp. & & & $\mathrm{x}$ & $\mathrm{x}$ & $\mathrm{x}$ & $\mathrm{x}$ \\
\hline 8 Ruptitermes sp. & $\mathrm{x}$ & $\mathrm{x}$ & $\mathrm{x}$ & $\mathrm{x}$ & $\mathrm{x}$ & \\
\hline 9 Tetimatermes sp. & $\mathrm{X}$ & $\mathrm{x}$ & $\mathrm{x}$ & $\mathrm{x}$ & $\mathrm{x}$ & $\mathrm{x}$ \\
\hline 10 Diversitermes sp. & & & $\mathrm{x}$ & & & \\
\hline \multicolumn{7}{|l|}{ RHINOTERMITIDAE } \\
\hline 11 Heterotermes longiceps (Snyder, 1924) & & & $\mathrm{x}$ & $\mathrm{x}$ & $\mathrm{x}$ & $\mathrm{x}$ \\
\hline \multicolumn{7}{|l|}{ TERMITIDAESyntermitinae } \\
\hline 12 Labiotermes laticephalus Silvestri, 1901 & $\mathrm{x}$ & $\mathrm{x}$ & $\mathrm{x}$ & $\mathrm{x}$ & & \\
\hline 13 Labiotermes longilabius Silvestri, 1901 & & & & $\mathrm{x}$ & & \\
\hline 14 Labiotermes orthocephalus Silvestri, 1901 & & & & $\mathrm{x}$ & & \\
\hline 15 Procornitermes triacifer Silvestri, 1901 & $\mathrm{x}$ & $\mathrm{x}$ & $\mathrm{x}$ & $\mathrm{x}$ & $\mathrm{x}$ & $\mathrm{x}$ \\
\hline 16 Rhynchotermes diphyes Mathews, 1977 & $\mathrm{x}$ & & $\mathrm{x}$ & & $\mathrm{x}$ & \\
\hline 17 Syntermes nanus Constantino, 1995 & $\mathrm{x}$ & $\mathrm{x}$ & $\mathrm{x}$ & $\mathrm{x}$ & & \\
\hline 18 Syntermes molestus (Burmeister, 1839) & $\mathrm{x}$ & $\mathrm{x}$ & $\mathrm{x}$ & $\mathrm{x}$ & $\mathrm{x}$ & $\mathrm{x}$ \\
\hline
\end{tabular}

As espécies do grupo 2 (H. longiceps, L. laticephalus e S. nanus) e do grupo 4 (Grigiotermes sp. (7), Ruptitermes sp. (8), Tetimatermes sp. (9) e Rhynchotermes diphyes (16) foram mais específicas com os tratamentos GH2 e SH2, respectivamente. O grupo 3 esteve representado somente por Syntermes molestus (18), espécie que estava associada aos tratamentos MH1 e MH2 (Figura 1).

Na ocorrência periódica das espécies de cupins nos meses e nos sistemas de manejo da irrigação, destacaram-se Anoplotermes sp. 1, quanto à frequência relativa no sistema de gotejamento; e S. molestus, no sistema de microaspersão e gotejamento, ambas em março de 2012 (Tabela 2).

Anoplotermes sp. 1, Anoplotermes sp. 2 e $S$. molestus foram coletados em todos os meses. Anoplotermes sp. 3 ocorreu em 10 meses, enquanto Labiotermes orthocephalus e Diversitermes sp., apenas em janeiro e fevereiro de 2013, respectivamente.
Labiotermes longilabius foi coletado nesses dois meses. Ruptitermes sp. foi coletado em março, abril e junho de 2012.

A relação entre as variáveis pluviosidade, umidade relativa e temperatura com as espécies de cupins foi estabelecida em duas componentes ortogonais pela $\mathrm{ACP}$, que permitiu identificar a relação entre características extraídas das variáveis de forma independente em relação ao objeto cupim. Pode-se conhecer a influência de fatores ambientais sobre a comunidade de cupins ocorrentes em plantas de eucalipto submetidas a diferentes formas de irrigação.

De acordo com os vetores de cada variável em cada componente, foi possível designar o primeiro eixo como a componente do efeito da pluviosidade e umidade relativa, uma vez que os pesos de intensidade para esses fatores foram elevados, atingindo escores de 0,968 e 0,721 , respectivamente. Conforme o 
Tabela 2 - Frequência relativa (FR) total de espécies de cupins em área cultivada com eucaliptos, híbridos grancam e urograndis, nos sistemas de manejo de irrigação, no período de março de 2012 a fevereiro de 2013, no Município de Aquidauana, MS.

Table 2 - Relative frequency (RF) total termite species in an area cultivated with eucalyptus, hybrid grancam and urograndis, in irrigation management systems, from March 2012 to February 2013, in the municipality of Aquidauna, MS.

\begin{tabular}{|c|c|c|c|c|}
\hline \multirow{2}{*}{ Espécies } & \multicolumn{3}{|c|}{ Tratamento } & \multirow{2}{*}{$\% \mathrm{FR}$} \\
\hline & Microaspersão & Gotejamento & Sequeiro & \\
\hline Anoplotermes sp. 1 & 47 & 124 & 33 & 24,03 \\
\hline Anoplotermes sp. 2 & 31 & 78 & 29 & 16,25 \\
\hline Anoplotermes sp. 3 & 3 & 8 & 4 & 1,77 \\
\hline Anoplotermes sp. 4 & 3 & 11 & 3 & 2,00 \\
\hline Anoplotermes sp. 5 & 7 & 2 & 9 & 2,12 \\
\hline Aparatermes sp. & 7 & 10 & 8 & 2,94 \\
\hline Grigiotermes sp. & 1 & 5 & 4 & 1,18 \\
\hline Ruptitermes sp. & 2 & 3 & 2 & 0,82 \\
\hline Tetimatermes sp. & 5 & 7 & 5 & 2,00 \\
\hline Diversitermes sp. & 0 & 1 & 0 & 0,12 \\
\hline Heterotermes longiceps & 2 & 7 & 2 & 1,30 \\
\hline Labiotermes laticephalus & 4 & 6 & 10 & 2,36 \\
\hline Labiotermes longilabius & 0 & 1 & 0 & 0,12 \\
\hline Labiotermes orthocephalus & 0 & 2 & 0 & 0,24 \\
\hline Procornitermes triacifer & 10 & 9 & 9 & 3,30 \\
\hline Rhynchotermes diphyes & 2 & 3 & 1 & 0,71 \\
\hline Syntermes nanus & 11 & 15 & 26 & 6,12 \\
\hline Syntermes molestus & 134 & 112 & 31 & 32,63 \\
\hline Freq. total & 269 & 404 & 176 & 100,00 \\
\hline
\end{tabular}

g.1 $=15 ;(\mathrm{p}<0)$; frequência relativa $(\mathrm{FR})$ mínima de 20.1 .

g.1 =15; $(p<0)$; relative frequency $(F R)$ minimum 20.1 .

posicionamento das espécies (objetos) na figura bidimensional (Figura 2) e os pesos das variáveis nas componentes, foi possível caracterizar as relações da precipitação e umidade como intensas e diretamente proporcionais com $R$. diphyes e Ruptitermes sp. que tiveram, de modo geral, escores elevados no eixo 1. O efeito da pluviosidade e da umidade relativa foi caracterizado por escores inversos para as espécies Anoplotermes sp. 4, H. longiceps, Diversitermes sp. e Anoplotermes sp. 5. No eixo 1, a influência das variáveis precipitação e umidade relativa sobre a comunidade de cupins foi de $68,8 \%$. As espécies mais influenciadas foram R. diphyes, Anoplotermes sp. 5, Diversitermes sp., Grigiotermes sp., H. longiceps, Anoplotermes sp. 4 e Ruptitermes sp., respectivamente, em ordem decrescente.

O segundo componente (eixo 2) limitou-se essencialmente à temperatura ambiente, uma vez que apenas essa variável teve peso de intensidade de $-0,762$. O primeiro eixo teve elevada consistência interna, com á $=0,688$, enquanto no eixo 2 a consistência interna foi reduzida, com $\alpha=0,04$. As espécies Aparatermes sp. (6), Grigiotermes sp., Tetimatermes sp. e $L$. longilabius tiveram escores positivos e mais distantes da origem dos eixos, respectivamente em ordem decrescente, aumentando suas atividades com o incremento da temperatura até o limite de calor ideal para essas espécies. As espécies que tiveram escores negativos e distantes da origem dos eixos foram $S$. nanus, S. molestus e L. laticephalus. As espécies Anoplotermes sp. 1, Anoplotermes sp. 2, Anoplotermes sp. 3, L. orthocephalus e Procornitermes triacifer (15) foram indiferentes a pluviosidade, umidade relativa e temperatura, com escore próximo da origem dos eixos.

\section{DISCUSSÃO}

O ambiente de eucalipto submetido a diferentes tipos de irrigação permitiu a coleta de diversas espécies de cupim. O método utilizado na coleta de cupins pode influenciar a riqueza. Junqueira e Berti Filho (2000) obtiveram somente quatro gêneros em levantamento de cupins em área de plantação inicial de eucalipto com o uso de armadilhas Termitrap ${ }^{\circledR}$. Esse número reduzido possivelmente ocorreu pelo fato de o material 


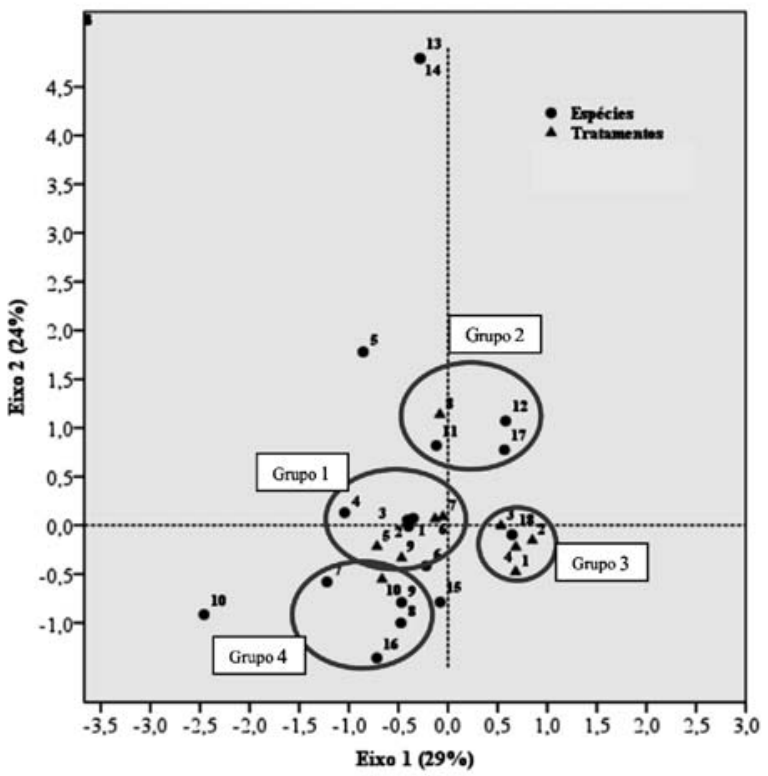

Figura 1-Análise de correspondência entre espécies de cupins em área cultivada com eucaliptos, híbridos grancam e urograndis, nos sistemas de manejo de irrigação, no período de março de 2012 a fevereiro de 2013, no Município de Aquidauana, MS.

Figure 1-Analysis of correspondence between termite species in an area cultivated with eucalyptus, hybrids grancam and urograndis, in irrigation management systems, from March 2012 to February 2013, in the municipality of Aquidauna, MS.

utilizado na elaboração dessas armadilhas exercer atratividade para número restrito de espécies. Com o método de amostragem de solo, em parcelas distribuídas em transecto, Varma e Swaran (2007) coletaram 11 espécies de cupim pertencentes a duas famílias e quatro subfamílias. Esses autores atribuíram a alta diversidade de espécies à eficiência do protocolo de amostragem de solo em parcelas. Sales et al. (2010), com o uso do mesmo método em área de reflorestamento com o eucalipto, obtiveram 21 espécies, representando duas famílias e 16 gêneros. Portanto, possivelmente o elevado número de espécies com diversos hábitos tróficos obtidos neste estudo pode ser explicado pelo intenso esforço amostral (1.719 amostras) pela eficiência do método utilizado e, também, pela peculiaridade ambiental do ecótono Cerrado/ Pantanal.

Entre as espécies coletadas, houve alta frequência relativa de Syntermes molestus (Termitidae: Syntermitinae) e Anoplotermes sp. 1 (Termitidae: Apicotermitinae). Espécies de Anoplotermes ocorreram em vários tipos

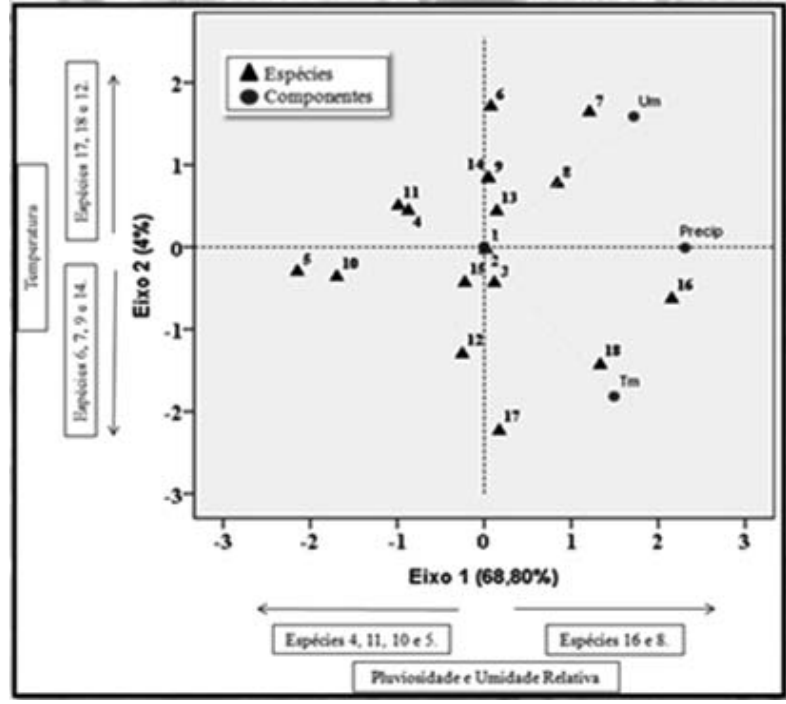

Figura 2 - Análise de componentes principais da relação entre a pluviosidade, umidade relativa, temperatura e as espécies de cupins em área cultivada com eucaliptos, híbridos grancam e urograndis, nos sistemas de manejo de irrigação, no período de março de 2012 a fevereiro de 2013, no Município de Aquidauana, MS.

Figure 2 - Principal Components Analysis of the relation between rainfall, relative humidity, temperature and species of termites in an area cultivated with eucalyptus, hybrid grancam and urograndis, in irrigation management systems, from March 2012 to February 2013, in the municipality of Aquidauna, MS.

de hábitats, e a maioria vive em galerias difusas no solo, alimentando-se de matéria orgânica em decomposição (SALES et al., 2010). O gênero Syntermes é considerado praga do eucalipto e, neste estudo, ocorreu em todos os tratamentos. Junqueira et al. (2008) citaram que $S$. molestus atacou plantas de até 1 ano de idade, danificando a raiz e o coleto. $\mathrm{O}$ gênero Heterotermes também tem sido registrado na literatura como responsável pelo dano em mudas de eucalipto. Neste estudo, $H$. longiceps foi coletado, porém com baixa frequência. Calderon e Constantino (2007) verificaram danos de H. longiceps em raízes de mudas jovens de Eucalyptus urophylla. Nesta pesquisa, quando da implantação das mudas no campo, estas foram severamente atacadas por cupins, o que implicou a realização de replantio. Possivelmente, as espécies S. molestus e H. longiceps foram responsáveis pela morte da maior parte das mudas de eucalipto, podendo-se inferir que esses insetos já estavam estabelecidos no solo quando as mudas foram plantadas. 
A riqueza obtida em levantamentos em uma área, além de ser afetada pela antropização, pode variar em função das castas obtidas nas amostragens, uma vez que na maioria das espécies a identificação somente é possível pela morfologia dos soldados. Neste estudo, em muitas amostras foram obtidos apenas operários e não foi possível identificar o gênero e as espécies às quais pertenciam. Esse fato, provavelmente, impediu que o número de espécies obtidas fosse superior. Possivelmente, os pontos de amostragens sem soldados coincidiram com setores dos túneis ocupados somente pelos operários, como também os soldados podem ter-se deslocado em galerias mais profundas, em níveis inferiores ao do perfil do solo amostrado.

Não foi verificado um padrão de distribuição de ocorrência das espécies de cupim durante o ano de execução deste experimento. Contrariamente, Almeida e Alves (2009), em Piracicaba, SP, verificaram que a atividade de forrageamento de Heterotermes tenuis em cultura de cana-de-açúcar teve picos marcados de ocorrência com máximos de janeiro a abril e de zero de junho a agosto, atribuindo essa variação aos fatores climáticos. Essa afirmação está de acordo com as características climáticas relatadas por Sanzigolo (2008) para Piracicaba, onde, historicamente, ocorre máxima de $38{ }^{\circ} \mathrm{C}$ em janeiro e mínima de $-0,1{ }^{\circ} \mathrm{C}$ em julho.

A falta de um padrão definido na distribuição das espécies de cupins durante o ano pode ser atribuído ao clima da região deste estudo que se caracteriza por invernos moderados, com $19,3^{\circ} \mathrm{C}$ de temperatura mínima média histórica em julho e máxima média de $25,6^{\circ} \mathrm{C}$ em janeiro (ZARONI et al., 2011). Esses valores climáticos são considerados adequados para a atividade normal dos cupins. Gallo et al. (2002) citaram que a temperatura ótima para o desenvolvimento de espécies de cupim é de $25^{\circ} \mathrm{C}$. A $38^{\circ} \mathrm{C}$, tem-se a temperatura limiar máxima (base superior) e a $15^{\circ} \mathrm{C}$, a limiar mínima (base inferior). Dentro desse intervalo, encontram-se a faixa ótima de desenvolvimento e a atividade dos indivíduos.

Houve diversidade de comportamento das espécies de cupim diante do teor de umidade do solo nos diferentes tratamentos deste estudo. As espécies que tiveram baixa frequência nas coletas foram mais específicas por determinados tratamentos, observando-se que pequenas mudanças ambientais causaram fortes efeitos na ocorrência desses insetos (Figura 1).
$\mathrm{Na}$ análise de correspondência, a maior afinidade pelo tratamento de microaspersão observada em $S$. molestus corrobora outros estudos, que permitiram comprovar a atratividade diferenciada de algumas espécies de cupim por teores elevados de umidade no solo. Gautam (2011) referiu que a umidade e a temperatura desempenham papel vital e influenciam o forrageamento e a desidratação desses insetos. Em experimentos com Coptotermes formosanus, Shiraki (1909) constatou que essa espécie teve adaptação ampla na faixa de umidade, menos em areia seca e saturada. A relação entre umidade e taxa de tunelamento de duas colônias de C. formosanus foi estudada por Campora e Grace (2009) em condições de laboratório. Embora a quantidade de água incorporada na arena de ambas as colônias fosse a mesma, houve diferenças na taxa de tunelamento. Esses autores deixaram evidente que existem necessidades distintas no teor de umidade em populações diferentes, ainda que sejam da mesma espécie. Portanto, a presença de S. molestus no sistema de microaspersão, em que o solo recebeu a maior quantidade de água na irrigação, pode indicar que essa espécie apresenta maior afinidade com a água no perfil do solo. A diversidade de espécies encontradas nos sistemas de gotejamento e de sequeiro possivelmente implica diferente preferência entre essas espécies por menores teores de umidade no solo.

As precipitações pluviométricas ocasionaram diferentes respostas às espécies de cupins nesta pesquisa. O fato de que R. diphyes e Ruptitermes sp. tiveram aumento populacional diretamente proporcional à ocorrência de chuvas pode ter ocorrido, provavelmente, porque essas espécies apresentam maior afinidade por solo mais úmido ou por terem diferente capacidade de tunelamento.

A afinidade de cupins por alta umidade no solo foi verificada por Almeida e Alves (2009) em H. tenuisna cultura da cana-de-açúcar, em que o aumento progressivo das precipitações elevou o número de cupins coletados em armadilhas. Para Dibog et al. (1998), o provável efeito-chave das precipitações pluviométricas e outros fatores ambientais sobre os cupins tem sido escassamente abordados. A forte correlação encontrada por esses autores entre a abundância e a riqueza e o total da precipitação pluviométrica acumulada em dois dias anteriores à amostragem fizeram sugerir que as diferenças nessas variáveis foram devidas às mudanças na distribuição dos cupins no perfil do solo em curto espaço 
de tempo, provavelmente para escapar da inundação vertical através das galerias.

A diminuição de populações de cupins imediatamente após intensas precipitações foi observada neste estudo, permitindo inferir que os insetos aprofundaram os túneis subterrâneos até camadas inferiores menos saturadas. Existem marcadas diferenças de tolerância de diversas espécies de cupins quando da inundação do solo, como demonstrado por Forschler e Henderson (1995), em ensaios de imersão dos cupins em água, encontrando o menor tempo letal médio $\left(\mathrm{TL}_{50}\right)$ e $\left(\mathrm{TL}_{90}\right)$ de 11,1 e 15,8 horas para Coptotermes formosanus. Reticulitermes flavipes (Kollar, 1837) foi o mais tolerante, com $\mathrm{TL}_{50}$ de $19,6 \mathrm{~h} \mathrm{e} \mathrm{TL}_{90}$ de 29,7 h. Quando os ensaios foram realizados em arenas, a sobrevivência foi maior pelo fato de que nas galerias dos cupins permaneceram pequenos espaços com ar que permitiram a sobrevivência dos insetos, embora houvesse encharcamento do ambiente. As espécies de cupins que foram coletadas neste trabalho, ainda após fortes precipitações, constroem ninhos difusos no solo. Essa característica provavelmente favoreceu a sua permanência na camada de amostragem por disporem de oxigenação suficiente no seu sistema de tunelamento. A correlação negativa entre Anoplotermes sp. 4, H. longiceps, Diversitermes sp. e Anoplotermes sp. 5 com a precipitação pluviométrica foi evidente durante as amostragens. A ocorrência acumulada de chuvas dos 30 dias anteriores à amostragem diminuiu a frequência dessas espécies. Esse fato deixa evidente a plasticidade das diversas espécies de cupins perante diferentes teores de umidade no perfil do solo.

A localização inicial de alimento em diversas espécies é realizada pelos soldados que logo incentivam os operários para realizar o tunelamento e coleta dos recursos. Konate et al. (2000) observaram em Macrotermes bellicosus (Smeathman, 1781) (Termitidae) que somente os soldados mais velhos acompanhavam os operários nas viagens de forrageamento, enquanto os mais jovens permaneciam no ninho. Santos (2008) verificou que cada uma das diversas espécies estudadas respondeu, de forma diferente, às alterações ambientais impostas pela mudança nas estações, e essas respostas estão associadas às características de cada uma delas, tal como o tamanho corporal. A diferença no tamanho é fator-chave para determinação dos diferentes padrões temporais na atividade de forrageamento.
Além do tamanho corporal, os fatores climáticos podem afetar a taxa de produção de soldados e operários, como comprovado por Sattar et al. (2013), que obtiveram correlação não significativa entre precipitação e porcentagem de operários em Microtermes obesi Holmgren 1911; no entanto, houve correlação positiva e significativa da chuva sobre proporção de operários em Odontotermes lokanandi Chatarjee e Thakur, 1967. Santos (2008) observou que a ocorrência de Coptotermes gestroi teve correlação negativa com a umidade do solo e Heterotermes longiceps, correlação positiva. Portanto, as variações de coleta das espécies neste trabalho podem ter sido devidas a diferentes proporções de indivíduos que refletiram nas amostras contendo soldados, como também ao comportamento diferenciado de preferência diante de variações dos teores de umidade do solo.

A pouca influência exercida pela temperatura sobre a comunidade de cupins observada difere dos resultados obtidos por Arab e Costa-Leonardo (2005), que observaram efeito da temperatura no comprimento dos túneis construídos por Coptotermes gestroi e Heterotermes tenuis. O comprimento dos túneis secundários aumentou significativamente a $15^{\circ} \mathrm{C}$ nessas duas espécies. Sattar et al. (2013) observaram correlação positiva entre a temperatura atmosférica e operários de Microtermes obesi (Holmgren, 1913) e correlação não significativa entre a temperatura atmosférica e operários de Odontotermes lokanandi. Portanto, as observações diferentes na atividade dos cupins nesta pesquisa e as daqueles autores não devem ser consideradas discrepantes, mas podem significar uma característica de plasticidade de adaptação entre as espécies de cupim e a temperatura de cada local, o que provavelmente influenciou a quantidade de espécies amostradas.

\section{CONCLUSÕES}

A região de transição Cerrado/Pantanal apresenta riqueza de cupins em área de cultivo com eucalipto, que é influenciado pelos diferentes manejos da irrigação e pela precipitação pluviométrica e umidade relativa do ar.

\section{REFERÊNCIAS}

ALMEIDA, J. E. M.; ALVES, S. B. Atividade de forrageamento de Heterotermes tenuis (Hagen) (Isoptera: Rhinotermitidae) em cana-de-açucar utilizando a armadilha Termitrap ${ }^{\circledR}$. Arquivo Instituto Biologico, v.76, n.4, p.613-618, 2009. 
ANDRADE, L. R. M. Corretivos e fertilizantes para culturas perenes e semiperenes. In: SOUSA, D. M. G.; LOBATO, E. Cerrado, correção do solo e adubação. 2.ed. Brasília: Embrapa, 2004. p.317-366.

ARAB, A.; COSTA-LEONARDO, A. M. Effect of biotic and abiotic factors on the tunneling behavior of Coptotermes gestroi and

Heterotermes tenuis (Isoptera: Rhinotermitidae). Behavioural Processes, v.70, n.1, p.32-40, 2005.

CALDERON, R. A.; CONSTANTINO, R. A Survey of the termite fauna (Isoptera) of na eucalipt plantation in central Brazil.

Neotropical Entomology, v.36, n.3, p.391395, 2007.

CAMPORA, C. E.; GRACE, J. K. Comparison of tunneling in the laboratory and field by the formosan subterranean termite, Coptotermes formosanus (Isoptera: Rhinotermitidae).

Sociobiology, v.53, n.2b, p.389-401, 2009.

DIBOG, L.; EGGLETON, P.; FORZI, F.

Seasonality of soil termites in a humid tropical forest, Mbalmayo, southern Cameroon.

Journal of Tropical Ecology, v.14, p.841-850, 1998.

FORSCHLER, B. T.; HENDERSON, G.

Subterranean termite behavioral reaction to water and survival of inundation: implication for field populations. Environnental

Entomology, v.24, n.6, p.1592-1597, 1995.

GALLO, D.; NAKANO, O.; SILVEIRA NETO, S.; CARVALHO, R. P. L.; BAPTISTA, G. C. de; BERTI FILHO, E.; PARRA, J. R. P.; ZUCCHI, R. A.; ALVES, S. B.; VENDRAMIM, J. D.; MARCHINI, L. C.; LOPES, J. R. S.; OMOTO, C. Entomologia agrícola, Piracicaba: FEALQ, 2002.920p.

GAUTAM, B. K. Role of substrate moisture, relative humidity and temperature on survival and foraging behavior of formosan subterranean termites. (Ph.D.) Louisiana State University, Baton Rouge. 2011. Disponivel em: < http:// etd.lsu.edu/docs/available/etd-10302011-181759> Acesso em: 15 jun. 2013.
HAMMER, O.; HARPER, D. A. T.; RYAN, P. D. PAST version 3.0: Paleontological Statistics Software Package for Education and Data Analysis. Palaeontologia Electronica, 4:9. 2013.

JONES, D. T.; EGGLETON, P. Sampling termite assemblages in tropical forests: testing a rapid biodiversity assessment protocol. Journal of Applied Ecology, v.37, n.1, p.191-203, 2000.

JUNQUEIRA, L. K.; BERTI FILHO, E. Termites (Insecta: Isoptera) in plantings of Eucalyptus spp. (Myrtaceae) in Anhembi, State of São Paulo, Brazil. Acta Biologica Leopoldensia, v.22, n.2, p.205-211, 2000.

JUNQUEIRA, L. K.; DIEHL, E.; BERTI FILHO, E. Termites in eucalyptus forest plantations and forest remmants: an ecological approach.

Bioikos, v.22, n.1, p.3-14, 2008.

KONATE, S.; LEUTHOLD, R.; HARI, M.; VEIVERS, P. Colour variation and polyethismo of the soldier caste in the termite Macrotermes bellicosus. Entomologia Experimentalis et Applicata, v.94, n.1, p.51-55, 2000.

MARoco, J. Análise estatística com utilização do SPSS. 3.ed. Lisboa: Silabo, 2007. 822p.

NAIR, K. S. S.; VARMA, R. V. Some ecological aspects of the termite problem in young eucalypt plantations in Kerala, India. Forest Ecology and Manegement, v.12, n.3-4, p.287-303, 2003.

OLIVEIRA, D. E.; QUEIROZ, L. L.; BERGAMINI, L. L.; FALEIRO, F. A. M. V.; ANJOS, L.; SANTOS, T.; BRANDÃO, D. Diversidade de cupins (Insecta: Isoptera) no cerrado do parque estadual da Serra de Caldas Novas, Goiás, Brasil. In: CONGRESSO BRASILEIRO DE ENTOMOLOGIA, 22., 2008, Uberlândia-MG. Anais... Uberlândia: 2008. Disponível em: http://www.seb.org.br/eventos/ SBE/XXIICBE/resumos/R0253-1.html> Acesso em: 28 dez. 2012.

PAES, J. B. Resistência natural da madeira de Corymbia maculata (Hook.) K.D.Hill e L.A.S. Johnson a fungos e cupins xilófagos, em condições de laboratório. Revista Árvore, v. 26, n. 6, p. 761-767, 2002.

Revista Árvore, Viçosa-MG, v.39, n.1, p.137-146, 2015 
PERALTA, R. C. G.; MENEZES, E. B.; CARVALHO, A. G.; AGUIAR-MENEZES, E. L. Wood consuption of forest species by subterranean termites (Isoptera) under field conditions.

Revista Árvore, v.28, n.2, p.283-289, 2004.

SALES, M. J. D.; MATOS, W. C.; REIS, Y. T.; RIBEIRO, G. T. Frequência e riqueza de cupins em áreas de plantio de eucalipto no litoral norte da Bahia. Pesquisa Agropecuária

Brasileira, v.45, n.12, p.1351-1356, 2010.

SANZIGOLO, C. A. Distribuição de extremos de precipitação diária, temperatura máxima e mínima e velocidade do vento em Piracicaba, SP (19172006). Revista Brasileira de Meteorologia, v.23, n.3, p.341-346, 2008.

SANTOS, A. F.; AUER, C. G.; GRIGOLETTI JÚNIOR, A. Doenças do eucalipto no sul do Brasil: identificação e controle. Colombo: Embrapa, 2001. (Circular Técnica)

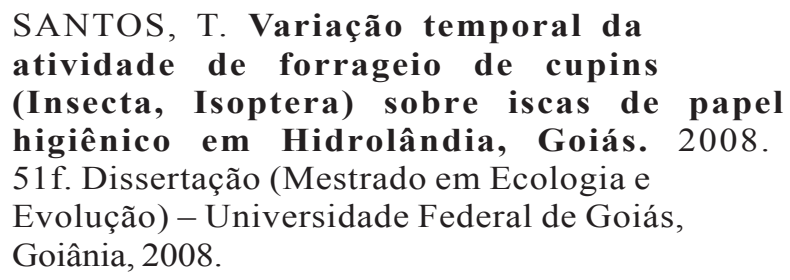

Revista Árvore, Viçosa-MG, v.39, n.1, p.137-146, 2015
SATTAR, A.; NAEEM, M.; UL-HAQ, E. Impact of environmental factors on the population dynamics, density and foraging activities of Odontotermes lokanandi and Microtermes obesi in Islamabad. Springer Plus, v.2, p.349-355, 2013.

SILVA, J. C.; LOPEZ, A. G. C.; OLIVEIRA, J. T. $\mathrm{S}$. Tree age influence on Eucalyptus grandis wood natural resistance to deterioration by dry-wood termites. Revista Árvore, v. 28 n.4, p.1-7, 2004.

VARMA, R. V.; SWARAN, P. R. Diversity of termites in a young eucalypt plantation in the tropical forest of Kerala, India.

International Journal of Tropical Insect Science, v.7, n.2, p.95-101, 2007.

ZARONI, M. J.; AMARAL, F. C. S.; SILVA, E. F.; COELHO, M. R.; RIBEIRO, W. C.;

BHERING, S. B.; CHAGAS, C. S.; PEREIRA, N. R.; GONCALVES, A. O.; DART, R. O.; AGLIO, M. L. D.; LOPES, C. H. L.; TAKAGI, J. S.; EARP, C. G. S. Zoneamento Agroecologico do Municipio de Aquidauana-MS - Dados eletronicos. Rio de Janeiro: Embrapa Solos, 2011.63p. (Boletim de Pesquisa e Desenvolvimento, 185). 